\title{
Mapeamento geológico utilizando a relação de Poisson entre os campos gravimétricos e magnetométricos
}

Santos, Renata de Sena, UFOPA; Martins, Cristiano Mendel, UFPA.

Copyright 2018, SBG - Sociedade Brasileira de Geofísica

Este texto foi preparado para a apresentação no VIII Simpósio Brasileiro de Geofísica, Salinópolis, 18 a 20 de setembro de 2018. Seu conteúdo foi revisado pelo Comitê Técnico do VIII SimBGf mas não necessariamente representa a opinião da Comite Técnico do VIII SimBGr, mas não necessariamente representa a opiniáo da para propósitos comerciais sem prévia autorização da SBGt.

\section{Resumo}

Desenvolvemos um método de interpretação automática para o mapeamento geológico utilizando a Relação de Poisson entre os campos gravimétricos e magnetométricos. Presumimos que diferentes unidades geológicas apresentam diferentes propriedades físicas, e que a estimação destas propriedades, bem como a da Razão de Poisson, permite mapeá-las. Para mapearmos a Razão de Poisson realizamos a filtragem transformando os campos e a inversão estimando as prioridades físicas sempre no domínio do espaço. Nas inversões, utilizamos os funcionais regularizadores: Suavidade Global e Variação Total. Aplicamos estes procedimentos a um conjunto de dados sintéticos e a metodologia mostrou-se efetiva para o mapeamento de unidades geológicas.

\section{Introdução}

A identificação e interpretação mesmo qualitativa das fontes causadoras de uma anomalia a partir de um sinal geofísico observado, exclusivamente da análise visual desta anomalia são, em muitos casos, complexas e pouco efetivas. Mesmo o uso do conjunto das características elencadas que descrevam os materiais rochosos não garante uma relação direta $e$ determinística com as anomalias nos sinais geofísicas, uma vez que as relações causais entre fonte e sinais observados não são desprovidas de ambiguidades. Assim, a utilização de mais de um campo físico para a interpretação pode diminuir o grau de ambiguidade. Por outo lado, nem sempre o geólogo consegue aferir diretamente as litologias, por estarem inacessíveis ou cobertas por geleiras, oceanos, densas florestas ou camadas intemperizadas. Uma alternativa é o uso de metodologias de investigação indireta que cubram essas áreas inacessíveis. Assim, muitos procedimentos para interpretação automática são ofertados aos intérpretes para subsidia-lo na tarefa de identificar, localizar e delinear as fontes geofísicas. Propomos uma solução, identificando e caracterizando áreas de interesse através dos campos potenciais, integrando dois tipos de dados, a gravimetria e a magnetometria, desde que cubram a área de interesse. A interpretação conjunta entre os campos gravimétricos e magnetométricos foi empregada primeiramente por Garland (1951) e por Bott (BOTT; INGLES, 1972). Apresentamos um método para mapear unidades geológicas pela integração de duas estimações distintas, obtendo como produtos diretos mapeamentos de densidade aparente e de susceptibilidade aparente de uma camada superficial, e como subprodutos, mapeamento envolvendo a razão entre estas propriedades físicas, chamada Razão de Poisson, que é estabelecia pela Relação de Poisson (1826), a qual incorpora para ser válida um conjunto de restrições a respeito das fontes e dos campos destes dois métodos. Para estimarmos parâmetros geofísicos a partir de métodos potenciais utilizamos técnicas de inversão acopladas a funcionais matemáticos regularizadores (BARBOSA; SILVA; MEDEIROS, 1999; LIMA et al. 2011; MARTINS et al., 2011; SILVA; COSTA; BARBOSA, 2006; SILVA; MEDEIROS; BARBOSA, 2001). Estas técnicas utilizam funcionais estabilizadores (TIKHONOV e ARSENIN, 1977) formulados de modo à simultaneamente garantir estabilidade à inversão e a incorporar informações a priori aos parâmetros estimados. A relação geofísica funcional entre os campos gravimétricos e magnetométricos e os parâmetros físicos causadores, é linear em ambos os casos. No entanto selecionamos dois diferentes funcionais estabilizadores, um dos quais tem relação não linear com os parâmetros. Assim, procedemos com dois tipos distintos de inversão, uma linear e outra não linear. Quanto a inversão linear, como etapas de processamento pretérito a obtenção do mapa da Razão de Poisson são indispensáveis, as procedemos aplicando 0 conceito de camada equivalente (DAMPNEY, 1969), que por um lado viabiliza a procedimento de filtragem no domínio do espaço, e por outro lado demanda uma inversão linear a fim de obter-se uma distribuição de fontes equivalentes. Assim, realizamos uma etapa de inversão linear seguida de outra etapa de inversão não linear, esta ultima demanda pelo tipo de regularizador adotado.

Realizamos um conjunto de teste em que procedemos às estimações das propriedades físicas $e$ as transformações nos campos para obtermos os mapas das Razões de Poisson, e assim caracterizamos a eficácia da inversão e dos mapeamentos na recuperação das informações geológicas simuladas.

\section{Metodologia}

\section{Modelo direto}

Discretizamos o conjunto de unidades geológicas lateralmente dispostas em uma camada superficial, finita no plano $x-y$, em prismas retangulares tridimensionais horizontalmente justapostos em uma malha de $m x-$ $m y$ de $M$ prismas, com topo plano e coincidente com a superfície $\left(z_{t}\right)$ e base paralela ao topo e profundidade constante $\left(z_{b}\right)$, em um sistema destral de coordenadas, como o eixo $Z$ positivo para baixo, e o eixo $X$ apontado para o Norte, como mostra a Figura 1. Presumimos que, dentro da mesma unidade geológica, a densidade e a intensidade de magnetização das rochas não variam. Por simplicidade utilizamos modelo interpretativo em que as coordenadas horizontais das observações são coincidentes com os centros horizontais dos prismas. Assim, para cada $i$-ésimo ponto de observação (nas coordenadas $\left.x_{i}, y_{i}, z_{i}\right)$, podemos escrever uma expressão genérica dos campos $f$ (gravimétrico e magnético) por:

$f\left(x_{i}, y_{i}, z_{i}\right)=C \mathrm{~K} \int_{V} \frac{\left(z_{i}-z^{\prime}\right)}{r^{3}} d v$, 
sendo $C$ uma constante intrínseca a cada um dos campos, e K uma função que define a propriedade física causadora do campo gravimétrico $\left(f^{g z}\right)$ ou magnético $\left(f^{M}\right)$ e $r$ a distância entre a fonte e o ponto de avaliação do campo $\left(r=\sqrt{\left(x_{i}-x^{\prime}\right)^{2}+\left(y_{i}-y^{\prime}\right)^{2}+\left(z_{i}-z^{\prime}\right)^{2}}\right)$. Assim, especificamente: para o campo gravimétrico $C$ é a constante gravitacional de Newton $(\gamma)$ e K é a densidade $(\rho)$; para o campo magnético $C$ é a constante de magnetização $\left(C_{m}\right)$ e a função $\mathrm{K}$ é o produto $M\left[\frac{\partial}{\partial \widehat{\alpha}} \frac{\partial}{\partial \widehat{\beta}}\right]$, sendo $M$ a intensidade de magnetização da fonte e $\alpha$ e $\beta$ as direções de magnetização da fonte e de avaliação do sensor, respectivamente.

Como discretizamos as fontes em prismas, utilizamos as soluções algébrica-algorítmica da integral da equação 1 para avaliação dos campos gravimétrico e magnético produzidos por prismas. Para o caso gravimétrico essa solução é dada por Plouff (1976), para o caso magnético a essa solução é dada por Bhattacharyya (1964).

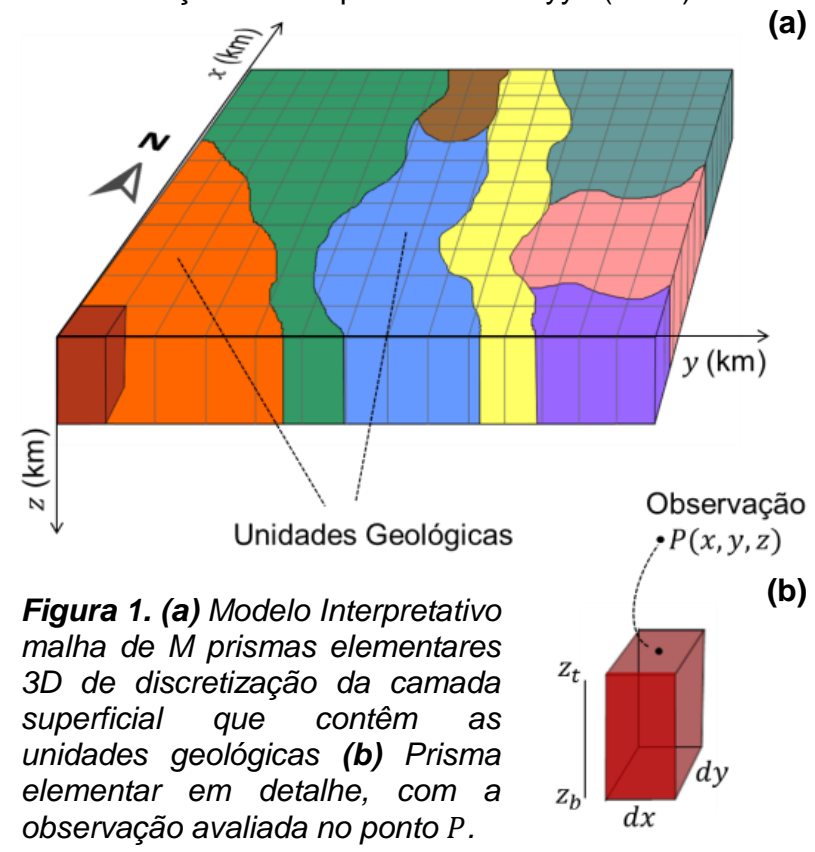

\section{Problema Inverso}

Formulamos e aplicamos dois tipos distintos de inversão, a linear a não linear para em ambos os procedimentos estimar uma distribuição de propriedade física em uma camada superficial. Empregamos na inversão linear dois funcionais, o ajuste dos dados e o regularizador dos parâmetros conhecido como regularizador de Tikhonov de primeira ordem (TWOMEY, 1963), denominado como vínculo de Suavidade Global (SG). Este funcional estabilizador consiste em minimizar a norma dois (norma Euclidiana) da aproximação por diferenças finitas da derivada horizontal de primeira ordem dos parâmetros, a minimização deste funcional favorece soluções de mapeamento associadas a distribuições de propriedades físicas de pontos vizinhos que apresentem valores próximos entre si.

Também empregamos na inversão não linear dois funcionais, o ajuste dos dados e o regularizador dos parâmetros, mas empregamos neste caso o regularizador de Tikhonov de primeira ordem (TWOMEY, 1963) minimizado via norma um (norma $L_{1}$ ). Este funcional regularizador é chamado de Variação Total, VT, (RUDIN et al., 1992; ACAR e VOGEL, 1994, MARTINS, 2009), por favorecer a estimativa de uma distribuição parâmetros que apresente descontinuidades abruptas. Para o caso de mapeamento de propriedades físicas, presumivelmente entre unidades geológicas distintas devem ocorrer descontinuidades abruptas dos valores dos parâmetros que as caracterizem, sejam eles a densidade ou a intensidade de magnetização.

Seja $\boldsymbol{Y}^{\boldsymbol{o}} \equiv\left(Y^{o}{ }_{1}, \cdots, Y^{o}{ }_{M}\right)^{T}$ um vetor $M$-dimensional contendo as observações dos campos adquiridos, gravimétrico ou magnético, presumivelmente produzidas pela camada superficial que contém as unidades geológicas a serem mapeadas. Os parâmetros a serem estimados (vetor $\boldsymbol{p}$ contendo as propriedades físicas da camada superficial em $M$ pontos discretos de uma malha $m_{x} \times m_{y}$ ) estão relacionados aos dados observados para o caso gravimétrico ou magnético pela relação linear mostrada na equação 1 . O problema inverso linear de estimar $\boldsymbol{p}$ a partir dos dados observados pode ser formulado como um problema de minimização, em relação a $\boldsymbol{p}$, do funcional

$\phi_{Y}=\frac{1}{M}\left\|\boldsymbol{Y}^{o}-\boldsymbol{Y}^{c}\right\|_{2}^{2}$

em que $\|\cdot\|_{2}$ é a norma Euclidiana e $\boldsymbol{Y}^{c}$ é um vetor $M$ dimensional cujo $i$-ésimo elemento contém o valor da observação teórica computada no $i$-ésimo ponto de observação utilizando-se a distribuição da propriedade física estimada $(\boldsymbol{p})$ para a camada. Para obtermos uma estimativa $(\boldsymbol{p})$ única e estável procuramos uma solução que simultaneamente produza ajuste aceitável ao dado observado $\left(\boldsymbol{Y}^{0}\right)$ e incorpore as informações exigidas por um funcional regularizador que simultaneamente introduz estabilidade e informação a priori sobre a distribuição dos parâmetros a serem estimados. Para tanto, formulamos o problema inverso linear de estimar os $M$ valores de propriedade física através da minimização de

$\phi_{S G}=\|\boldsymbol{R} \boldsymbol{p}\|_{2}$

sujeito a

$\phi_{Y}=\varepsilon^{2}$.

No funcional $\phi_{S G} \boldsymbol{R}$ é uma matriz $L \times M$ representando o operador diferencial discreto de primeira ordem ao longo das direções $x$ e $y$. O funcional $\phi_{S G}$ é o regularizador de Tikhonov de primeira ordem (TIKHONOV; ARSENIN, 1977) minimizado via norma Euclidiana denotada por $\|\cdot\|_{2}$. Devido ao produto $\boldsymbol{R p}$ ser uma aproximação por diferenças finitas da primeira derivada de $\boldsymbol{p}$ ao longo das direções $x$ e $y$, então através da minimização de $\|\boldsymbol{R} \boldsymbol{p}\|_{2}$, ficam favorecidas as soluções que são relativamente suaves e, implicitamente, é introduzida a informação geológica de que a distribuição da propriedade física é globalmente suave (BARBOSA et al., 1997). O vetor $\boldsymbol{p}$, contendo as $M$ estimativas dos valores da propriedade física, que soluciona o problema de otimização vinculado dado pela minimização do funcional 03 sujeito a explicar os dados geofísicos (condição 04), será obtido minimizando-se o funcional não vinculado:

$\lambda_{S G}(\boldsymbol{p})=\phi_{Y}+\mu_{S G} \phi_{S G}$

em que, $\mu_{S G}$ é o parâmetro de regularização, um escalar positivo que representa um peso associado ao funcional $\phi_{S G}$ e é dependente do tipo de propriedade física em que a inversão é aplicada.

A partir deste funcional não vinculado $\lambda_{S G}(\boldsymbol{p})$, podemos escrever 0 estimador que determina 0 vetor de parâmetros $\boldsymbol{p}$,

$\boldsymbol{p}=\left(\boldsymbol{A}^{T} \boldsymbol{A}+\mu_{S G} \boldsymbol{R}^{T} \boldsymbol{R}\right)^{-1} \boldsymbol{A}^{T} \boldsymbol{Y}^{o}$,

em que, $\boldsymbol{A}$ é a matriz sensibilidade, cujo $i j$-ésimo elemento é 
$\boldsymbol{A} \equiv\left\{a_{i j}\right\}=\frac{\partial f_{i}}{\partial p_{j}}$

sendo $f_{i}$ o funcional geofísico definido pelo campo a ser utilizado na inversão, especificamente podendo ser os campos definidos na equação 01.

Esta inversão formulada até aqui é linear, no entanto, se minimizarmos o funcional regularizador dos parâmetros utilizando a norma $l 1$

$\phi_{V T}=\|\boldsymbol{R} \boldsymbol{p}\|_{1}$,

formulamos o funcional chamado de Variação Total, que é não linear por conta da derivada, em relação aos parâmetros, deste funcional ser função dos parâmetros. Portanto buscamos resolver o problema inverso não vinculado de minimizando o funcional

$\lambda_{V T}(\boldsymbol{p})=\phi_{Y}+\mu_{V T} \phi_{V T}$,

em que, $\mu_{V T}$ é o parâmetro de regularização da Variação Total.

Para resolver iterativamente este problema não linear, empregamos o método de Gauss-Newton com a estratégia de Marquardt (1963). Neste procedimento, primeiro expande-se a função-objeto $\left(\lambda_{V T}(\boldsymbol{p})\right)$ em série de Taylor em torno da solução estimada $\boldsymbol{p}_{k}$ obtida na $k$ ésima iteração, mantendo-se apenas os termos de segunda ordem. Em seguida, deriva-se a função $\lambda_{V T}\left(\boldsymbol{p}_{k}+\Delta \boldsymbol{p}_{k}\right)$ em relação a $\Delta \boldsymbol{p}_{k}$ e iguala-se ao vetor nulo. Assim, a equação normal para a estimativa $\Delta \boldsymbol{p}_{k}$ é dada por:

$H_{k} \Delta \boldsymbol{p}_{k}=-J_{k}$,

em que $J_{k}$ e $H_{k}$ são, respectivamente, o vetor Jacobiano e a matriz Hessiana do funcional $\lambda_{V T}(\boldsymbol{p})$ avaliados na $k$ ésima iteração. A fim de evitar instabilidades na inversão, utilizamos uma aproximação da matriz Hessiana proposta por Martins (2009). A cada iteração o vetor de parâmetros $\boldsymbol{p}_{k}$ é atualizado pelo vetor de correções $\Delta \boldsymbol{p}_{k}$ que é somado ao vetor de parâmetros da iteração anterior $\boldsymbol{p}_{k-1}$

$\boldsymbol{p}_{k}=\Delta \boldsymbol{p}_{k}+\boldsymbol{p}_{k-1}$.

Embora a relação funcional entre os parâmetros e as observações seja linear (equações 01) o funcional $\phi_{Y}$ é atualizado iterativamente, pois $\boldsymbol{Y}_{k}^{c}$ depende de $\boldsymbol{p}_{k}$

$\boldsymbol{Y}_{k}^{c}=\boldsymbol{A p}_{k}$,

sendo $A$ a matriz de sensibilidade, de dimensões $M \times M$. $\mathrm{Na}$ primeira iteração, quando $k=1$, utilizamos como aproximação inicial $\left(\boldsymbol{p}_{0}\right) \quad 0$ vetor de parâmetros estimados pela inversão linear com suavidade global.

O algoritmo de inversão é interrompido quando não há mais significativa variação do funcional $\lambda_{V T}(\boldsymbol{p})$. Na prática a convergência ocorre quando, em uma $k$-ésima iteração, a seguinte inequação é satisfeita:

$\left|\frac{\lambda_{V T}\left(\boldsymbol{p}_{k}\right)-\lambda_{V T}\left(\boldsymbol{p}_{k-1}\right)}{\lambda_{V T}\left(\boldsymbol{p}_{k-1}\right)}\right| \leq 0.001$.

\section{Filtragem}

\section{Campos transformados no Domínio do Espaço}

Uma vez obtida as distribuições de propriedades físicas da camada superficial, as aplicamos no sentido do teorema da camada equivalente (DAMPNEY, 1969) para obtermos os campos transforados com procedimentos de filtragem no domínio do espaço (EMILIA, 1973, SILVA, 1986 e SILVA, 1996) e estes campos filtrados são utilizados na Relação de Poisson. Construímos três filtros através da multiplicação de uma matriz de transformação $(\boldsymbol{Q})$ pelos parâmetros estimados $(\boldsymbol{p})$. Uma vez estimada esta distribuição da propriedade física $(\boldsymbol{p})$, o campo transformado $\left(f_{T}\right)$ pode ser então obtido, como regra geral, por

$f_{T}=\boldsymbol{Q p}$,

sendo $\boldsymbol{Q}$ a matriz de transformação associada à filtragem a ser produzida, e cada elemento desta Matriz $M \times M$ contém as funções de Green (EMILIA, 1973) correspondente a transformação. Para obtermos a matriz de transformação do campo cada $i j$ - ésimo elemento da matriz $\boldsymbol{Q}$ é dado pela aplicação analítica da operação desejada na função do campo na $i j$ - ésima relação fonte-ponto de observação. Portanto, para calcularmos cada $i j$-ésimo elemento da matriz de transformação: i) $\boldsymbol{Q}^{g z z}$ para o gradiente vertical do campo gravimétrico basta aplicar o gradiente com respeito $z\left(\nabla_{z}\right)$ da componente vertical da anomalia gravimétrica; ii) $\boldsymbol{Q}^{\boldsymbol{R} \boldsymbol{P}}$ para a redução ao polo basta manipular a expressão da anomalia de campo total impondo-se que as direções $\hat{\alpha}$ e $\hat{\beta}$ sejam verticais (SILVA, 1986); iii) $\boldsymbol{Q}^{V I}$ para a integral vertical do campo magnético basta integrar com respeito a $z$ a anomalia magnética impondo-se o campo no polo. Especificamente:

i) $Q_{i j}^{g_{z z}}=\gamma \sum_{k=1}^{2} \sum_{l=1}^{2} \sum_{m=1}^{2} \mu_{k l m}\left[\arctan \frac{x_{k} y_{l}}{z_{m} r_{k l m}}\right]$;

ii) $Q_{i j}^{R P}=\frac{z_{2}}{r_{2}^{2}}-\frac{z_{1}}{r_{1}^{2}}$;

iii) $Q_{i j}^{V I}=\frac{2 z}{r^{2}}$

Em que: $x_{1}=\left(x_{i}-x_{j}\right)-d x / 2, \quad x_{2}=\left(x_{i}-x_{j}\right)+$ $d x / 2, y_{1}=\left(y_{i}-y_{j}\right)-d y / 2, y_{2}=\left(y_{i}-y_{j}\right)+d y / 2$, $\left(x_{i}, y_{i}, z_{i}\right)$ as coordenadas do $i$-ésimo ponto de observação, $\left(x_{j}, y_{j}\right)$ as coordenadas horizontais do centro da $j$-ésima fonte da camada equivalente; $z_{1}$ e $z_{2}$ a profundidade do topo e da base, respectivamente, da $j$-ésima fonte, $\mu_{k l m}=(-1)^{k}(-1)^{l}(-1)^{m}$ e $\quad r_{k l m}=$ $\left(x_{k}^{2}+y_{l}^{2}+z_{m}^{2}\right)^{1 / 2} ; r_{1}$ e $r_{2}$ a distância entre a $i$-ésima observação e o topo e a base da $j$-ésima fonte, respectivamente; $z$ a distância entre a $i$-ésima observação e o centro da $j$-ésima fonte.

A relação de Poisson, desde que as fontes obedeçam certas premissas, estabelece uma relação entre 0 potencial magnético $(W)$ e o potencial gravimétrico $(U)$ (GARLAND, 1951; POISSON, 1826),

$W(P)=-\frac{C_{m} \vec{M} \hat{\beta}}{\gamma \rho} \nabla U(P)$,

demanda que a razão entre a magnetização $(M)$ e a densidade $(\rho)$ seja constante, esta razão é comumente chamada de Razão de Poisson. A relação de Poisson mostra que o campo magnético $f^{M}$ e o gravimétrico $f^{g z}$ estão relacionados, sendo o gravimétrico uma ordem de derivação a menos que o magnético. No caso particular do campo magnético estar reduzida ao polo $\left(f_{R P}^{M}\right)$ e da integral vertical $\left(f_{V I}^{M}\right)$ ser aplica nestas condições, podemos escrever duas versões da relação de Poisson:

$\frac{f_{R P}^{M}}{f_{g z z}^{g z}}=C_{a} \frac{M}{\rho}$

e

$\frac{f_{V I}^{M}}{f^{g z}}=C_{b} \frac{M}{\rho}$

sendo $M$ e $\rho$ a intensidade de magnetização aparente a densidade aparente, respectivamente, estimadas para a camada equivalente e $C_{a}$ e $C_{b}$ são constantes que 
dependem das unidades dos campos e das propriedades físicas utilizadas. A equação 19 é utilizada por Garland (1951), e a equação 20 é mostrada por Salem et al. (2014). Neste ponto, fica claro o porquê de termos explicitado apenas os filtros para obtermos os campos transformados $f_{g z z}^{g z}, f_{R P}^{M}$ e $f_{V I}^{M}$.

Uma vez obtidas às distribuições das propriedades físicas $M$ e $\rho$ estimadas, as equações 19 e 20 mostram que podemos construir mais dois outros mapas além destes de $M$ e $\rho$, especificamente temos os mapas das razões entre os campos e/ou campos transformados e das constantes $C_{a}$ e $C_{b}$.

\section{Resultados}

Compulsoriamente presumimos algumas simplificações e/ou restrições ao problema abordado: i) as fontes das anomalias, seja gravimétrica ou magnética, estão confinadas a uma camada superficial com topo e base plano-paralelos; ii) os campos adquiridos são representativos apenas destas fontes rasas; iii) os contatos entre as unidades geológicas lateralmente dispostas são verticais; iv) a propriedade física é constante dentro de uma mesma unidade geológica.

Simulamos um modelo (Figura 2) contendo 5 unidades geológicas com duas diferentes distribuições de propriedade física, uma gravimétrica (distribuição de densidade), outra magnética (distribuição da intensidade de magnetização). No entanto ambas as distribuições de propriedade física contém apenas 4 unidades geológicas distintas. Assim, como o modelo tem 5 unidades geológicas, alguma unidade é apenas fonte gravimétrica e não magnética, e alguma unidade é apenas fonte magnética e não gravimétrica. As demais unidades são fontes de ambos os campos. As Figuras $2 \mathrm{a}$ e $2 \mathrm{c}$ mostram as duas diferentes distribuições de propriedades físicas. A densidade (Figura 2a) varia em 4 valores discretos: 200, 300, 400 e $700 \mathrm{~kg} / \mathrm{m}^{3}$. A intensidade de magnetização (Figura $2 \mathrm{c}$ ) varia em 4 valores discretos: $0.75,1.00,1.50$, e $2.00 \mathrm{~A} / \mathrm{m}$. A inclinação e a declinação das fontes (todos os prismas) e do campo principal têm mesma direção, assim: $i=I=20^{\circ}$ e $d=D=5^{\circ}$. A camada que contém o modelo simulado foi discretizada em uma malha regularmente espaçada de $40 \times 40$ prismas, de dimensões horizontais $\mathrm{dx}=\mathrm{dy}=1.0$ $\mathrm{km}$, dispostos ao longo das direções horizontais $\mathrm{x}$ e $\mathrm{y}$, sendo a direção $\mathrm{x}$ paralela à direção Norte. $\mathrm{O}$ topo dos prismas é posicionado na cota vertical $z_{t}=0.0 \mathrm{~km}$, e a base na cota $z_{b}=0.5 \mathrm{~km}$. As observações das anomalias gravimétricas e magnéticas foram realizadas nas mesmas coordenadas horizontais dos centros dos prismas, e na cota vertical $z_{o}=-1.0 \mathrm{~km}$.

Os campos calculados, gravimétrico (componente vertical) e magnético (anomalia de campo total) são mostrados, respectivamente, nas Figuras $2 \mathrm{~b}$ e $2 \mathrm{~d}$. Os dados sintéticos calculados (Figuras $2 b$ e $2 d$ ) foram contaminados pela realização de um ruído pseudoaleatório, de média nula e desvio padrão igual a $0.1 \mathrm{mGal}$ no caso gravimétrico e $0.5 \mathrm{nT}$ no caso magnetométrico, que são os ruídos instrumentais típicos presumidos. Para estas estimações utilizamos uma camada de mesma discretização do modelo direto, no entanto com espessura diferente, sendo $z_{t}=0.250 \mathrm{~km}$ e $\mathrm{z}_{\mathrm{b}}=0.500 \mathrm{~km}$. Isto se deve ao fato de atendermos a recomendações quanto à profundidade em que a camada equivalente deve ser posicionada (DAMPNEY, 1969). Em nossos testes verificamos que a espessura da camada deve ser pelo menos quatro vezes menor que o espaçamento horizontal das observações para não produzir ambiguidade.

Para as inversões gravimétricas utilizamos como dados de entrada apenas o sinal gravimétrico mostrado na Figura $2 b$.

Utilizamos na SG o parâmetro de regularização $\mu_{S G}=$ $1.10^{-4}$, e na VT também $\mu_{V T}=1 \cdot 10^{-4}$. Os mapas com a as distribuições de densidade estimada são: Figura $3 a$ estimado com a SG; Figura 3c estimado com a VT. A Figura $3 \mathrm{~b}$ mostra a anomalia gravimétrica ajustada, que é obtida pela avaliação gravimétrica produzida pelo mapa de densidade estimado pela SG, e a Figura 3d mostra o gradiente vertical da anomalia gravimétrica que é obtido pelo produto da matriz de transformação pelo vetor de parâmetros estimados da VT (equações 14 e 15).

Note que a inspeção dos mapas de densidade estimada (Figuras $3 a$ e 3c) mostra um bom delineamento dos contatos entre as quatro unidades geológicas de diferentes densidades, isso é fortuito uma vez que justamente o que queremos é utilizar estas distribuições de propriedade física estimadas para o mapeamento geológico. Chamamos a atenção para o fato de que, conforme o esperado, a unidade que apresenta uma variação na intensidade de magnetização, mas não apresenta variação na densidade (Figuras 2a e 2c), não é delineada somente com a estimativa da densidade.

Para as inversões magnetométricas utilizamos como dados de entrada apenas a anomalia de campo total mostrada na Figura 2d. A Figura 4 mostra o resultado destas inversões magnetométricas.

Os mapas com as distribuições de intensidade de magnetização estimada são: Figura $4 \mathrm{a}$ estimado com a SG; Figura 4c estimado com a VT. A Figura 4b mostra a anomalia de campo total ajustada, que é obtida pela avaliação magnética produzida pelo mapa de intensidade de magnetização estimado pela SG, a Figura 4d mostra a anomalia de campo total reduzida ao polo que é obtida pelo produto da matriz de transformação pelo vetor de parâmetros estimados da VT (equações 14 e 16) e a Figura 4e mostra a integral vertical da anomalia de campo total reduzida ao polo que é obtida pelo produto da matriz de transformação pelo vetor de parâmetros estimados da VT (equações 14 e 17). Note que a inspeção dos mapas de intensidade de magnetização estimada (Figuras 4a e 4c) mostra um bom delineamento dos contatos entre as quatro unidades geológicas de diferentes intensidades de magnetização. Novamente, chamamos a atenção para o fato de que, conforme o esperado, a unidade que apresenta uma variação na densidade, mas não apresenta variação na intensidade de magnetização (Figuras $2 \mathrm{a}$ e $2 \mathrm{c}$ ) não é delineada somente com a estimativa da intensidade de magnetização.

Novamente, comparando ambos os mapas de intensidade de magnetização estimada o obtido via VT é o que delimita em uma região mais confinada os contados entre as diferentes unidades.

Aplicamos os três filtros que se utilizam dos parâmetros estimados para, através da multiplicação pela matriz de transformação adequada, produzir os campos transformados utilizados nas Relações de Poisson. Validamos via comparação numericamente estes campos transformados pela avaliação do erro médio quadrático do resíduo entre o campo transformado via nosso procedimento e o campo transformado obtido pela formulação clássica da literatura utilizando fontes elementares pontuais (ponto de massa e dipolo). Como em todos os casos o erro médio quadrático foi 
virtualmente zero, consideramos correta a nossa aproximação, inclusive no caso específico do modelo apresentado nesta seção.

A fim de mapear todas as 5 unidades do modelo simulado, utilizamos ambos os campos e as Razões de Poisson mostradas nas equações 19 e 20.

As Figuras $5 a$ e $5 b$ mostram os mapas das razões de Poisson (k) entre as propriedades físicas estimadas (M e $\rho$ ) pela SG e VT, respectivamente. As Figuras 5c e 5d mostram as constantes $\mathrm{Ca}$ e $\mathrm{Cb}$ das equações 19 e 20, respectivamente.

Observe que a inspeção dos mapas das Razões de Poisson (Figuras $5 \mathrm{a}$ e $5 \mathrm{~b}$ ) mostra o excelente delineamento dos contatos entre as cinco unidades geológicas. Isto é ainda mais fortuito uma vez que finalmente mapeamos todas as cinco unidades do modelo. No entanto, a Razão de Poisson que utiliza as propriedades físicas estimadas $(M$ e $\rho$ ) pela VT delimita os contatos entre as unidades em uma região ainda mais confinada. Por outro lado os mapas das constantes $\mathrm{Ca}$ e $\mathrm{Cb}$ conseguiram delinear muito bem as cinco unidades geológicas do modelo. No entanto, ambos os mapas das constantes são virtualmente idênticos, apresentando diferença numérica ínfima.

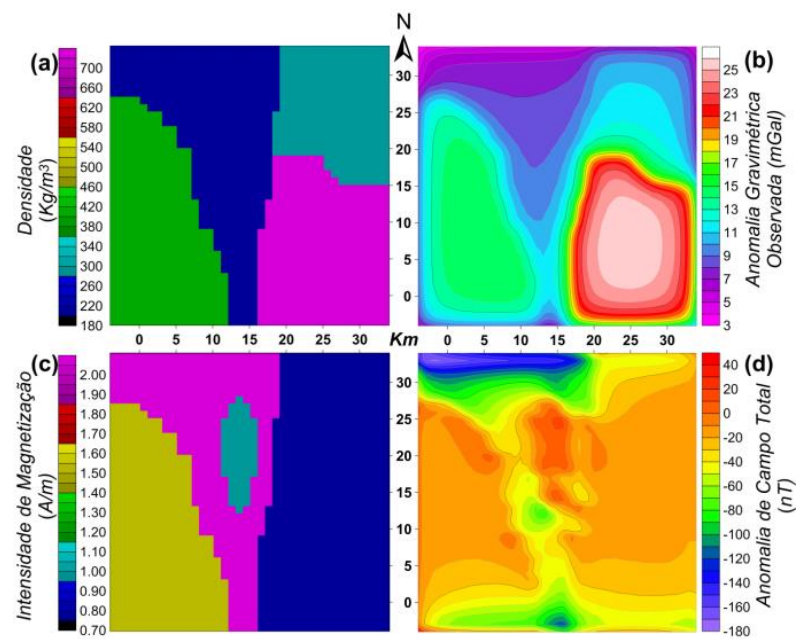

Figura 2 - Modelo Sintético Simulado - (a) distribuição de densidade $\rho$; (b) componente vertical da anomalia gravimétrica $f^{g z} ;(c)$ distribuição de intensidade de magnetização $M ;(d)$ anomalia de campo total $f^{M}$.

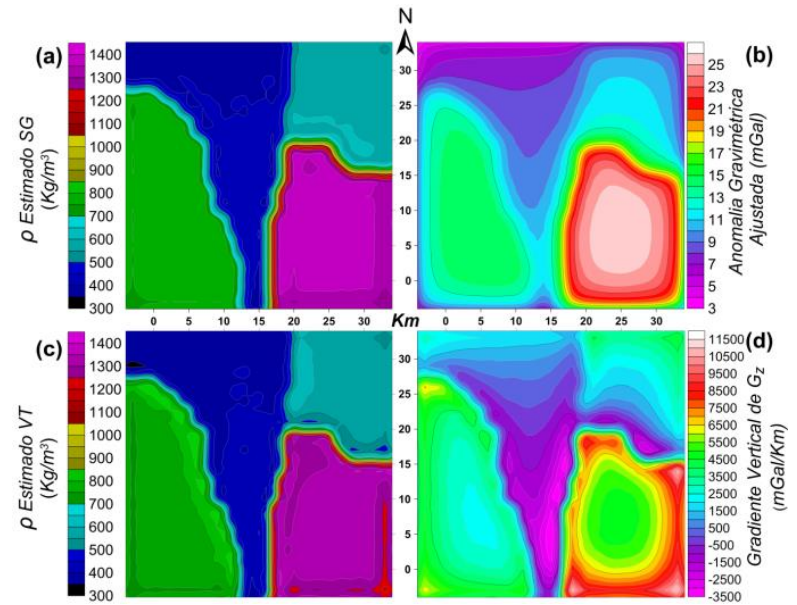

Figura 3 - Inversões dos dados gravimétricos - (a) densidades ( $\rho$ ) estimadas pela SG; (b) anomalia gravimétrica ajustada $\left(f^{g z}\right)$ pela $S G ;(c)$ densidades estimadas pela VT; (d) gradiente vertical de $\left(f_{g z z}^{g z}\right)$ pela VT.
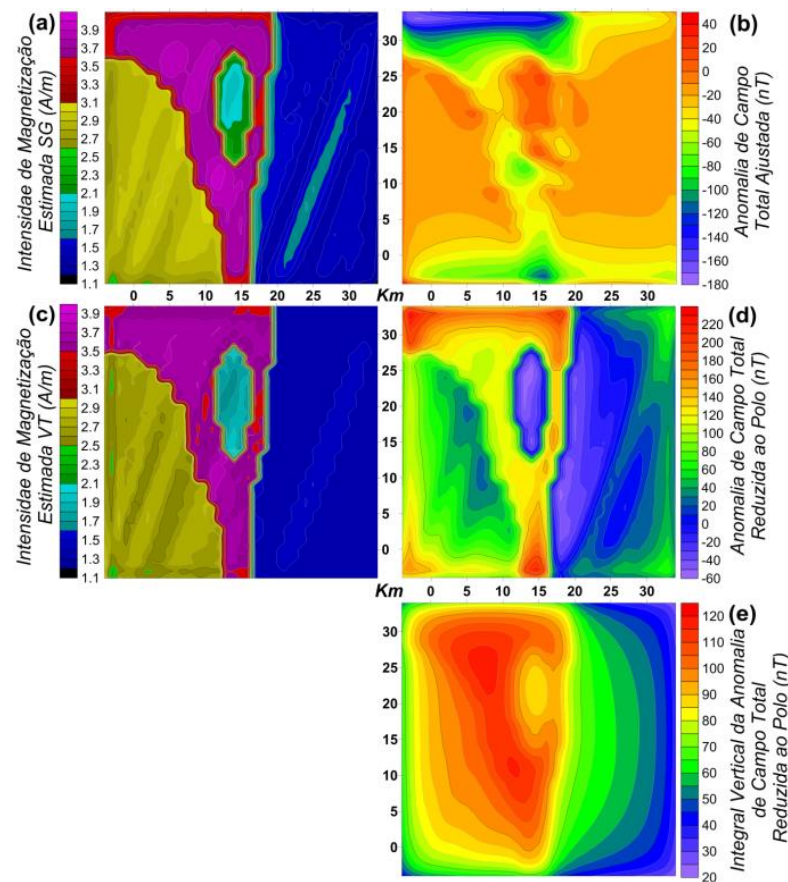

Figura 4 - Mapas das Inversões dos dados magnetométricos - (a) mapa das distribuições de intensidade de magnetização estimada pela SG; (b) mapa da anomalia de campo total ajustada obtida pela SG; (c) mapa das distribuições de intensidade de magnetização estimada pela VT; (d) mapa da anomalia de campo total reduzida ao polo; (e) mapa da Integral Vertical da anomalia reduzida ao polo.

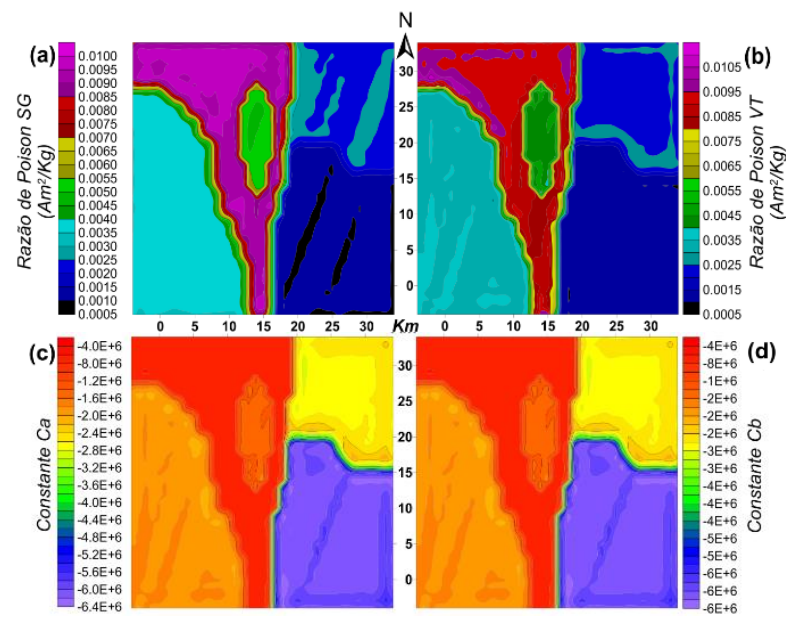

Figura 5 - Mapas com as Razões de Poisson - (a) mapa da razão de Poisson entre as propriedades físicas estimadas pela SG; (b) mapa da razão de Poisson entre as propriedades físicas estimadas pela VT (c) constantes $C_{a}$ da equação 19; (d) constantes $C_{b}$ da equação 20.

\section{Conclusões}

Apresentamos dois métodos de inversão, SG e VT aplicados em duas possibilidades de estimativa, para a densidade e para a intensidade de magnetização. Em seguida, utilizamos estas propriedades físicas estimadas para adicionalmente e via Relação de Poisson produzir mapas efetivos para fins de mapeamento geológico.

No entanto, no caso particular de alguma unidade não apresentar contraste de uma das propriedades físicas mapeadas, o mapeamento dos subprodutos da Relação 
Poisson que propomos, é efetivo mesmo para localizar e delinear tal unidade. Assim, a Relação de Poisson é uma janela para obter matematicamente a união entre os mapeamentos das diferentes fontes estimadas a partir de diferentes campos.

No desenvolvimento, construímos e aplicamos três filtros que se utilizam dos parâmetros estimados para, através da multiplicação pela matriz de transformação adequada, produzir os campos transformados necessários a serem utilizados nas Relações de Poisson, Gradiente Gravimétrico Vertical, Anomalia de Campo Total Reduzida ao Polo e a Integral Vertical do Campo Total Reduzido ao Polo. Durante os testes, verificamos que alguns cuidados são necessários na escolha da geometria da camada equivalente para não ocasionarmos problemas nos campos transformados.

Simulamos um modelo geológico apresentando diferentes unidades, e calculamos os correspondentes campos gravimétricos, magnetométricos e os campos transformados. No entanto, algumas unidades simuladas são fontes exclusivamente de apenas um dos campos. Utilizamos ambos os procedimentos de inversão, Suavidade Global e Variação Total, e ambos foram efetivos para mapear a distribuição da propriedade física fonte.

Mostramos os mapas produzidos a partir das Relações de Poisson e os mapeamentos das Razões de Poisson e das constantes destas Relações de Poisson se mostram efetivos para mapear todas as unidades do modelo. A metodologia proposta pode ser aplicada a dados reais, e como os mapeamentos obtidos prescindem de informações a priori, e dada a atual cobertura global de dados satelitais gravimétricos e magnetométricos, esta metodologia pode ser aplicada em regiões inacessíveis.

Como sugestões futuras, propomos estimar diretamente as Razões de Poisson das razões entre os campos transformados explicitadas das Relações de Poisson, preferencialmente utilizando a Inversão Variação Total.

\section{Agradecimentos}

Agradecimentos à Capes, ao CPGF-UFPA, ao laboratório de Métodos Gravimétricos e Magnetométricos (GMAG), e à UFOPA.

\section{Referências}

ACAR, R.; VOGEL, C. R. Analysis of bounded variation penalty methods for ill-posed problems. Inverse Problems, v. 10, p. 1217-1229. 1994.

BARBOSA, V. C. F.; SILVA, J. B. C.; MEDEIROS, W. E.Gravity inversion of basement relief using approximate equality constraints on depths. Geophysics, v. 62, p. 1745-1757. 1997.

BARBOSA, V. C. F.; SILVA, J. B. C.; MEDEIROS, W. E. Gravity inversion of a discontinuous relief stabilized by weighted smoothness constraints on depth. Geophysics, n. 64, p. 1429-1438. 1999.

BHATTACHARYYA, B. K., 'Magnetic anomalies due to prism-shaped bodies with arbitrary polarization', Geophysics, 29, 517-31. 1964.

BOTT, M. H. P.; INGLES, A. Matrix method for joint interpretation of two-dimensional gravity and magnetic anomalies with application to the Iceland-Faeroe Ridge: Geophys. J. Roy. Astr. Soc. 30, 1972. p. 55-67.

DAMPNEY, C.N.G. The Equivalent Source Technique. Geophysics, v. 34, n. 1, 1969.
EMILIA, D.A. Equivalent sources used as an analytic base processing total magnetic field profi lest. Geophysics, vol. 38, n. 2, 1973. p. 339-348.

GARLAND, G. D. Combined analysis of gravity and magnetic anomalies. SEG. University of Toronto, Toronto, Ontario, and St. Louis University, St. Louis, Missouri,1951.

LIMA, W. A.; MARTINS, M. C.; SILVA, J. B. C.; BARBOSA, V. C. F. Total variation regularization for depth-to-basement estimate: Part 2 - Physicogeologic meaning and comparisons with previous inversion methods. Geophysics, v. 76, n. 1, p. 113-120. 2011.

MARQUARDT, D. W. An algorithm for least-squares estimation of nonlinear parameters. Journal of the Society of Industrial and Applied Mathematics, v. 2, p. 601-612. 1963.

MARTINS, C. M. Inversão gravimétrica do relevo 3D de bacias sedimentares e da variação da densidade usando informação a priori sobre o ambiente geológico. 2009. 117f. Tese (Doutorado) - ON, 2009.

MARTINS, C. M.; LIMA, W. A.; BARBOSA, V. C. F.; SILVA, J. B. C. Total variation regularization for depthto-basement estimate: Part 1 - mathematical details and applications. Geophysics, v. 76, n. 1, 2011.

PLOUFF, D. Gravity and magnetic fields of polygonal prisms and application to magnetic terrain corrections. Geophysics, vol. 41. n. 4, 1976. p. 727-741.

Poisson, S. D. "Mémoire sur la Théorie du Magnétisme," Mémoires de l'Académie royale des Sciences de I'Institut de France, V (1826) 247-338.

RUDIN, L. I.; OSHER, S.; FATEMI, E. Nonlinear total variation based noise removal algorithms. Physica $D$, v. 60, p. 259-268, 1992

SALEM, A.; GREEN, C.; CHEYNEY, S.; FAIRHEAD, J. D.; ABOUD, E.; CAMPBELL, S. Mapping the depth to magnetic basement using inversion of pseudogravity: Application to the Bishop model and the Stord Basin, northern North Sea. seg.org. 2014.

SILVA, J. B. C. Reduction to the pole as an inverse problem and its application to low-atitude anomalies. Geophysics, vol. 51, n. 2,1986. p. 369-382.

SILVA, J.B.C. 2-D magnetic interpretation using the vertical integral. Geophysics, vol. 61 , n. 2, 1996. p. 387393.

SILVA, J. B. C.; MEDEIROS, W. E.; BARBOSA, V. C. F. Potential field inversion: choosing the appropriate technique to solve a geologic problem. Geophysics, v. 66 , n. 2, p. 511-520. 2001

SILVA, J. B. C; COSTA, D. C. L; BARBOSA, V. C. F. Gravity inversion of basement relief and estimation of density contrast variation with depth. Geophysics. v.71. n.5. p.151-158. 2006.

TIKHONOV, A. N.; ARSENIN, V. Y. Solutions of illposed problems. Washington: V.H. Winston \& Sons, 1977. 258p.

TWOMEY, S. On the numerical solution of Fredholm integral equations of the first kind by the inversion of the linear system produced by quadrature. Journal of the Association for Computing Machinery, v.10, p. 97-101. 1963. 